\title{
Robotic Dual-Console Distal Pancreatectomy: Could it be Considered a Safe Approach and Surgical Teaching even in Pancreatic Surgery? A Retrospective Observational Study Cohort
}

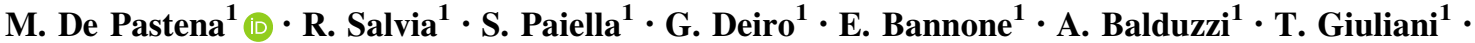 \\ L. Casetti $^{1}$ - M. Ramera $^{1}$ - C. Filippini $^{1}$ - G. Montagnini ${ }^{1}$ - L. Landoni $^{1}$ - A. Esposito ${ }^{1,2}$
}

Accepted: 10 June 2021 / Published online: 24 July 2021

(C) The Author(s) 2021

\begin{abstract}
Background The study aims to assess the safety and feasibility of the robotic dual-console during a robotic distal pancreatectomy

Methods The data of the consecutive patients submitted to RDP from 2012 to 2019 at the Verona University were retrieved from a prospectively maintained database. The patients submitted to RDP were divided into the dualconsole platform group (DG) and compared to the standard robotic procedure group (SG).

Results In the study period, 102 robotic distal pancreatectomies were performed, of whom 42 patients $(41 \%)$ belonged to the DG and 60 patients (59\%) to the SG. Higher operation time was recorded in the DG compared to the SG (410 vs. $265 \mathrm{~min}, p<0.001)$. The overall conversion rate of the series was 7\% (n 7 patients). All the conversions were observed in the SG $(p=0.021)$. No differences in morbidity or pancreatic fistula rate were recorded $(p>0.05)$. No mortality events in the 90th postoperative days were reported in this series.

Conclusions The robotic dual-console approach for distal pancreatectomy is safe, feasible, and reproducible. The postoperative surgical outcomes are comparable to the standard RDP with the single-console da Vinci Surgical System ${ }^{\circledR}$. This surgical technique can widely and safely improve the robotic surgical training program.
\end{abstract}

\section{Introduction}

The minimally invasive approach is obtaining wide popularity in pancreatic surgery, especially for the resections of the left part of the pancreas. Even if available in the literature only non-randomized studies comparing open resection with minimally invasive approaches, several benefits are reported about the latter's use, including less blood loss and shorter hospital stay [1-3]. Remarkably, the minimally invasive

A. Esposito

alessandro.esposito@aovr.veneto.it

M. De Pastena

m.depastena@gmail.com

1 Department of General and Pancreatic Surgery, The Pancreas Institute, University of Verona Hospital Trust, Verona, Italy

2 University of Verona, Verona, Italy approach to the distal pancreatic lesions is widely accepted when it is feasible as the gold standard for benign and uncertain behavior tumors [4, 5].

Despite its potential benefits, the technical limitations of conventional laparoscopy, such as rigid, non-articulated instruments and uncomfortable ergonomics, could preclude the diffusion and the implementation of a minimally invasive approach in pancreatic surgery. The introduction of robotic technology affords the surgeon to overcome the limitations of the conventional minimally invasive approach. Indeed, the da Vinci system (Intuitive Surgical, Sunnyvale, CA, USA) provided a better and magnified visualization of the surgical field with the 3D resolution, reduced natural tremors, increased surgical precision, and dexterity the introduction of the intraabdominal articulating instruments [6]. All these surgical and technical benefits seem to positively influence the clinical outcomes and reduce postoperative complications and hospitalization $[7,8]$. 
However, robot surgery emphasizes the single surgeon figure, which usually performs the procedure alone in the console. Unlike the laparoscopic or the open approach, the robotic procedure lost part of the benefits obtained from the assistant surgeons. This issue could be partially solved by the introduction of the da Vinci system dual-console. The use of the dual-console has shown advantages in reducing the learning curve and surgical training during different surgery $[9,10]$. To our knowledge, no data are reported in the literature regarding the use of the robotic dual-console in pancreatic surgery. Furthermore, no data are available to use, routinely, the dual-console, reproducing the steps and the movements of laparoscopic or open approach during pancreatic resection by expert surgeons, not only during the training program.

The study aims to assess the safety and feasibility of the robotic dual-console during a Robotic distal pancreatectomy (RDP).

\section{Materials and methods}

The study was performed according to the Strengthening the reporting of observational studies in epidemiology (STROBE) and Strengthening the reporting of cohort studies in surgery (STROCSS) guidelines [11, 12]. The Institutional Review Board's approval for data collection and analysis was obtained. Written informed consent was collected.

The consecutive patients submitted to RDP from 2012-2019 at the Verona University were retrieved from a prospectively maintained database.

Each patient was submitted preoperatively to a Contrastenhanced CT scan of the abdomen. All cases were preoperatively reviewed at a dedicated institutional surgical meeting, where the decision to perform a minimally invasive procedure was undertaken among staff surgeons. The Da Vinci Surgical System ${ }^{\circledR}$ Xi dual-console platform (Intuitive Surgical, Sunnyvale, CA) was used to perform the RDP from 2018. Indications for the use of the robot or the type of procedure were based on the availability of the Da Vinci Surgical System ${ }^{\circledR}$ and the surgeon's judgment, as previously described [13, 14]. Briefly, indications for a minimally invasive approach were benign or pre-malignant lesions smaller than $10 \mathrm{~cm}$ or, for malignancies, tumors without evidence of major vessel involvement. Additional resections, beyond cholecystectomy, adrenalectomy, or wedge resection of the stomach, were an exclusion criterion.

\section{Data collection and definitions}

Demographic and baseline characteristics consisted of sex, age, body mass index (BMI, $\mathrm{kg} / \mathrm{m}^{2}$ ), American society of anesthesiologists (ASA) physical status, and previous abdominal surgery.

The intraoperative data were composed of the type of procedure, conversion rate, operative time (minute), blood loss (milliliter), and pancreatic stump management (stapler or ultrasonic device).

All 90 day postoperative complications were scored and classified with the Clavien-Dindo system and the Comprehensive complication index [15]. The major complications as Clavien-Dindo grade III or higher. The postoperative pancreatic fistula was defined by the updated definition [16].

The length of stay (days), 90 day reoperation, readmission, and mortality rates were recorded. The final pathology, tumor size $(\mathrm{mm})$, and the number of lymph nodes harvested were also collected.

\section{Surgical procedure}

During the study period, the technique did not change regarding the steps of the procedure, the transection technique adopted, and the difficulty level. Briefly, the robotic docking was performed at the patient's head, which was in a supine position, at least $15 \mathrm{~cm}$ from the operating table. The open technique created a pneumoperitoneum at the umbilicus with $12 \mathrm{mmHg} \mathrm{CO} \mathrm{CO}_{2}$ pressure. Based on the different types of robots, the trocars were placed under visual control: the optical port at the umbilicus, two in the left hypochondrium, and one in the right hypochondrium. The assistant operative $12 \mathrm{~mm}$ port was positioned in a lower position between the umbilical and the first left port. The latter was usually used for the ultrasonic devices (HARMONIC FOCUS or ACE®; Johnson \& Johnson Medical, Ethicon, Somerville, NJ, USA), scissors, clip applicator, suction, and the eventual transection of the pancreas by stapler device. Robotic instruments, such as a grasper, bipolar coagulation, and monopolar hook were routinely used. Considering the indication for RDP, the pancreatic transection level and the splenic preservation were tailored. The intraoperative ultrasound was used to assist the surgical team in the selection of organ sparing procedures. The management of the pancreatic stump was reported previously [17], consisting of a stapler reinforced with a PGA felt $($ NEOVEIL $®$ Endo GIA $^{\mathrm{TM}}$ Reinforced Reload with Tri-Staple ${ }^{\mathrm{TM}}$ Technology $60 \mathrm{~mm}$; COVIDIEN, North Haven, CT, USA), or an ultrasonic dissector (HARMONIC FOCUS or ACE®; Johnson \& Johnson Medical, Ethicon, Somerville, NJ, USA). No additional sutures or patches were added during both techniques. The surgical field was drained by a surgical tube placed proximal to the pancreatic remnant. The postoperative drain management was based on the policy of early drain removed previously published [18]. 


\section{Minimally invasive surgeons' expertise and training strategy adopted}

The same two surgeons always performed the surgical procedures, which already had completed the learning curve in laparoscopic distal pancreatectomy. According to Napoli et al. [19], the leading senior surgeon completed the learning curve of RDP during the standard technique phase, while the trainee achieved this result during the dual-console phase. The assistant surgeons at the bedside were always experienced pancreatic surgeons that already completed the learning curve in laparoscopic distal pancreatectomy.

The Verona robotic training program was reserved for the surgeons that completed the laparoscopic distal pancreatectomy learning curve. The training program was divided into three different steps. Initially, all the trainees received the detailed technique description. The latter was composed of a checklist of the surgical instruments and a detailed procedure description and tips and tricks to prevent and solve potential intraoperative adverse events. Step one included the video training. Video training revised the entire procedure, and surgical tips and tricks were discussed with the senior surgeon. Step two was composed of the simulator training and cadaver lab. The robotic training followed the Intuitive Surgical recommendations and the official DaVinci surgical training. The third step was on-site proctoring by the senior surgeon.

\section{Statistical analysis}

Continuous variables were presented as means and standard deviation, or median and interquartile range, when pertinent. Student's t and Mann-Whitney U tests were used to compare continuous variables. Nonparametric tests were used when appropriate. Comparative analysis between groups was conducted using Fisher's exact tests for categorical variables. A $p$ value $<0.05$ was considered statistically significant (two-tailed). Data were analyzed using Statistical Package for the Social Sciences 25.0 (IBM Corp., Armonk, NY, USA).

Table 1 Demographic, intraoperative, and pathological data

\begin{tabular}{|c|c|c|c|c|}
\hline \multicolumn{5}{|l|}{ Study population $\mathrm{N}=102$} \\
\hline & Total n(\%) & Dual-console group $42(41 \%)$ & Standard group $60(59 \%)$ & $p$-value \\
\hline Age (years, DS) & $51 \pm 16$ & $51 \pm 16$ & $51 \pm 15$ & 0.810 \\
\hline Sex (Female) & $73(72 \%)$ & $28(67 \%)$ & $45(75 \%)$ & 0.243 \\
\hline BMI $\left(\mathrm{Kg} / \mathrm{m}^{2}, \mathrm{IQR}\right)$ & 23 [22-27] & 23 [20-29] & 23 [22-27] & 0.603 \\
\hline Previous abdominal surgery & $42(41 \%)$ & $20(48 \%)$ & $22(37 \%)$ & 0.184 \\
\hline ASA score I-II & $94(92 \%)$ & $37(88 \%)$ & $57(95 \%)$ & 0.183 \\
\hline Spleen preservation & $35(34 \%)$ & $17(41 \%)$ & $18(30 \%)$ & 0.188 \\
\hline Conversion & $7(7 \%)$ & $0(0 \%)$ & $7(12 \%)$ & 0.021 \\
\hline Pancreatic transection level & & & & 0.568 \\
\hline GDA & $2(2 \%)$ & $0(0 \%)$ & $2(3 \%)$ & \\
\hline Neck & $58(57 \%)$ & $25(60 \%)$ & $33(56 \%)$ & \\
\hline Distal & $42(41 \%)$ & $17(40 \%)$ & $25(41 \%)$ & \\
\hline Management stump & & & & $<0.001$ \\
\hline Stapler & $33(32 \%)$ & $23(55 \%)$ & $10(17 \%)$ & \\
\hline Ultrasonic scalpel & $67(66 \%)$ & $19(45 \%)$ & $48(80 \%)$ & \\
\hline Duration of surgery (minutes, IQR) & $337[260-401]$ & $410[330-480]$ & $265[230-330]$ & $<0.001$ \\
\hline EBL (cc, IQR) & $150[100-250]$ & $150[50-225]$ & $100[100-275]$ & 0.811 \\
\hline Pathology, No. (\%) & & & & 0.687 \\
\hline PDAC & $10(10 \%)$ & $5(12 \%)$ & $5(8 \%)$ & \\
\hline Cystic lesions & $28(27 \%)$ & $9(21 \%)$ & $19(32 \%)$ & \\
\hline pNET & $42(41 \%)$ & $18(43 \%)$ & $24(40 \%)$ & \\
\hline Other & $22(22 \%)$ & $10(24 \%)$ & $12(20 \%)$ & \\
\hline Tumor size (mm, DS) & $31 \pm 22$ & $30 \pm 20$ & $32 \pm 23$ & 0.661 \\
\hline Harvest lymph nodes (DS) & $15 \pm 12$ & $15 \pm 11$ & $14 \pm 12$ & 0.666 \\
\hline
\end{tabular}

Statistically significant values $(p<0.05)$ are given in bold

ASA: American society of anesthesiology; BMI: Body mass index; GDA: Gastroduodenal artery; MCN: Mucinous cystic neoplasm; pNET: Pancreatic neuroendocrine tumor; SPT: Solid pseudopapillary tumor 


\section{Timeline Recruitment}

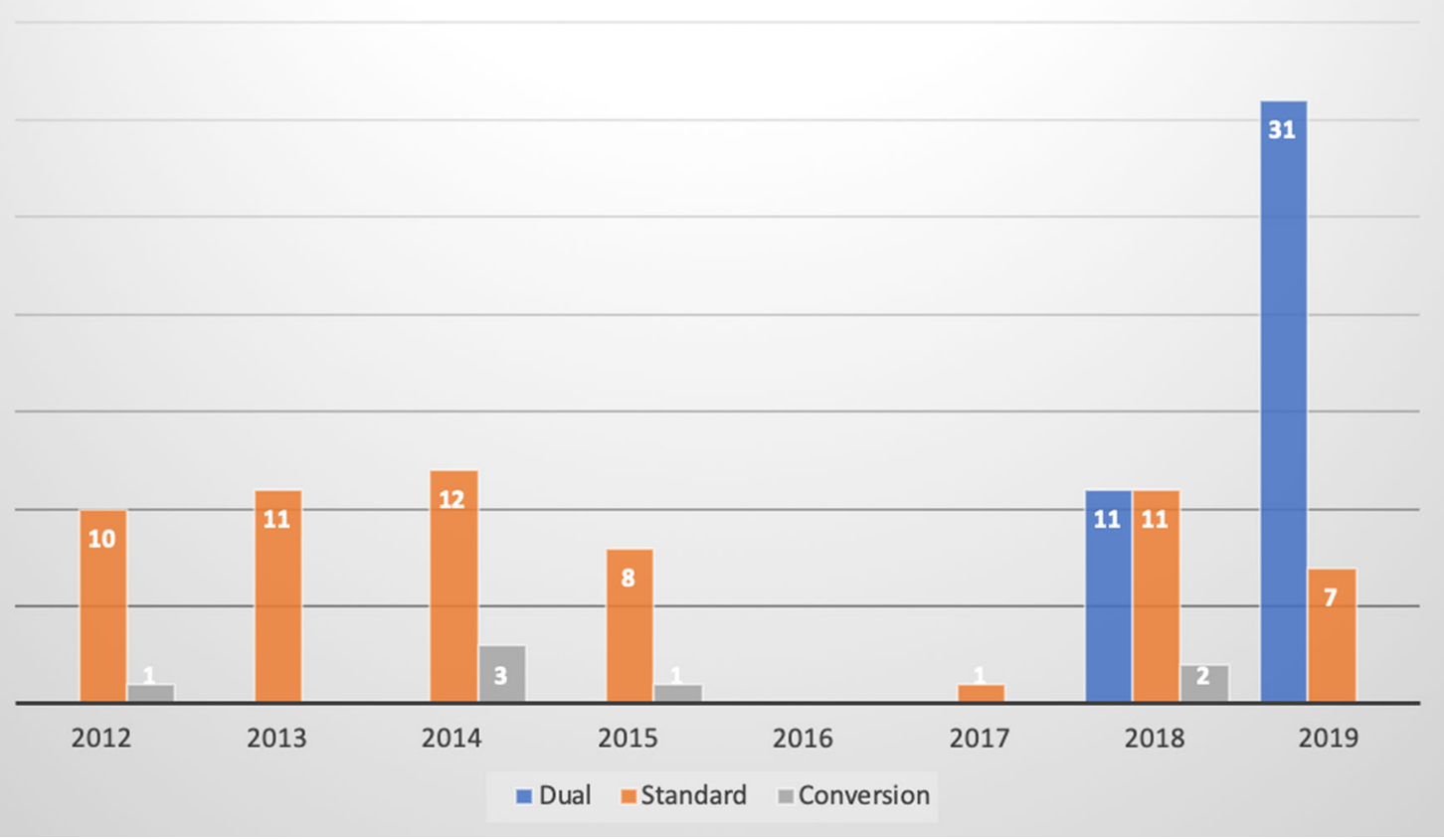

Fig. 1 Timeline recruitment

\section{Results}

In the study period, 102 robotic distal pancreatectomies were performed, of whom 42 patients $(41 \%)$ belonged to the Dual-console technique group (DG) and 60 patients (59\%) to the Standard procedure (SG). Demographic, intraoperative, and pathological data are shown in table 1. The baseline characteristics of the patients were balanced between groups. Higher operation time was recorded in the DG compared to the SG (410 vs. $265 \min , p<0.001)$. The overall conversion rate of the series was $7 \%$ (n 7 patients). All conversions were observed in the SG $(p=0.021)$. The approach to robotic distal pancreatectomy over time with the conversion rate is reported in Fig. 1. Moreover, different management of the pancreatic stump was recorded $(p<0.001)$. The ultrasonic scalpel was the most common device used in the SG (80\%). Otherwise, the stapler was frequently used during the dual-console technique (55\%). Notably, the two patients' pancreatic transection was performed by the knife associated with the stump oversewn.

Regarding the final pathology, the groups were homogenous $(p=0.687)$. No differences were found in the mean tumor size ( 30 vs. $32 \mathrm{~mm}$ ) and harvest lymph nodes (15 vs. 14$)$ ( $p=0.661$ and $p=0.666$, respectively).

Table 2 Postoperative data

\begin{tabular}{lllll}
\hline Study population $\mathrm{N}=102$ & & & & \\
\hline & Total $\mathrm{n}(\%)$ & Dual-console group 42 (41\%) & Standard group 60 (59\%) & $p$-value \\
\hline Overall morbidity & $44(43 \%)$ & $17(41 \%)$ & $27(45 \%)$ & 0.402 \\
Major complications & $10(10 \%)$ & $2(5 \%)$ & $8(13 \%)$ & $11 \pm 15$ \\
CCI & $10 \pm 14$ & $9 \pm 14$ & $16(27 \%)$ & 0.136 \\
POPF & $29(28 \%)$ & $13(31 \%)$ & $7(12 \%)$ & 0.631 \\
PPH & $9(9 \%)$ & $2(5 \%)$ & $7[6-10]$ & 0.199 \\
Length of stay (days, IQR) & $7[6-10]$ & $7[7-10]$ & $7(12 \%)$ & 0.618 \\
Reoperation & $8(8 \%)$ & $1(2 \%)$ & $10(17 \%)$ & 0.086 \\
Readmission & $12(12 \%)$ & $2(5 \%)$ & 0.060 \\
\hline
\end{tabular}

CCI: Comprehensive complication index; POPF: Postoperative pancreatic fistula; PPH: Post pancreatectomy hemorrhage 
Postoperative outcomes were outlined in table 2. The overall morbidity was $43 \%$ (n 44 patients) without difference between groups ( $41 \%$ vs. $45 \%, p=0.402)$. The postoperative pancreatic fistula occurred in 29 patients (28\%), similar between the two groups (31\% vs. $27 \%$, $p=0.400)$. Two grade $\mathrm{C}$ fistulae were detected in the SG. Five patients required a laparoscopic surgical revision. No statistical differences between groups were recorded $(5 \%$ vs. $12 \%, p=0.199)$. No mortality events in the 90th postoperative days were reported in this series.

\section{Discussion}

The robotic dual-console approach for distal pancreatectomy is safe and feasible. Even if there is an initial increase in the operation time, it does not negatively impact the clinical course in terms of postoperative outcomes. Indeed, the mortality nihil and no conversion were performed. The use of the dual-console da Vinci Surgical System ${ }^{\circledR}$ is not inferior compared to the standard RDP in a high volume of robot-assisted pancreatic surgery.

Minimally invasive robotic pancreatic surgery continues to gain popularity compared to laparoscopy due to improved patient outcomes, such as reduced hospital stay and faster recovery time [3]. RDP's learning process is still under investigation, varying from 10 to 40 cases to complete the learning curve [19-21]. Within a niche specialty, such as robotic pancreatic surgery, several years may be necessary to make the surgeon complete an RDP safely. However, rather than the time needed to attain it, it is fundamental to do it safely.

Trainees of robotic surgery should learn by doing rather than by observing [22]. Simulators or training platforms are helpful to familiarize with the technique and memorize all the procedures' steps. However, the introduction of the dual-console da Vinci Si Surgical System ${ }^{\circledR}$ allows an active and, at the same time, controlled training [23]. Indeed, it has been widely accepted that active trainee attendance during surgery on the robotic console is vital to improving the robotic curriculum [24].

The traditional surgical teaching model adopted for open surgery, "see one, do one, teach one", is hard to apply to robotic surgery, where, instead, the gradual transition of autonomy from attending surgeon to the trainee may be safely and quickly possible using the dual-console technique. The second console's introduction allows controlled and safe teaching, reproducing the open surgery steps and differing from laparoscopy. Furthermore, during the robotic procedure, the surgeon can learn more complex procedures step by step, offering the possibility to the proctor to activate, modify or improve the surgical act, like open surgery. The traditional surgical teaching model adopted for open surgery can be reviewed for robotic surgery in "see one, do side by side one, teach one."

This approach has already been investigated and validated in different abdominal surgeries and specialties $[9,25,26]$. These studies demonstrated that the dual-console robotic system could overcome defects of laparoscopic surgical training. The real essence of laparoscopic surgery is usually associated with an impression of distance and dissociation between the proctor and the trainee. During a laparoscopic procedure, the classical "4-handed" technique is usually uncomfortable due to the interference and conflict that could be generated by the laparoscopic instruments and camera and the surgeons. Furthermore, the continuous switching from the first operator function and the camera between proctor and trainee is a protracted, slow, and complicated movement during surgery. The exchange of the camera, which causes a temporary loss of the surgical vision, resetting the operative field to the original status, results in increased operative time, perseverance, and sometimes tolerance from the proctor. Additionally, the proctor's attention cannot always have the procedure under control increases anxiety and stress. All these issues may be solved by introducing the robotic dualconsole technique.

The study has some limitations that may prevent it from being generalized. First, the research was conducted on a retrospective basis. Therefore, the selection bias may be burdensome in choosing the type of surgery performed and even the robotic-assisted approach itself. Second, the sample size is limited, reducing the power of the statistical analysis. Third, the dual-console approach was introduced recently. This could possibly generate a selection bias. Fourth, the specific cost of this technology could be a limit to its diffusion. Has been widely described that the robotic technology has still high and important crude costs and its economic impact is debated yet [13]. Even if the literature reported promising surgical outcomes, the expenses of the robot application are reducing the possibility to perform surgical procedure and implement the surgical robotic training program. However, the diffusion of robotic technology could implement and improve this surgical approach, encouraging the introduction of the dual-console in a standardized training program.

The study results demonstrated the safety and the feasibility of the dual-console approach in pancreatic surgery. Future studies should prove it routinely useful.

\section{Conclusion}

The robotic dual-console approach for distal pancreatectomy is safe, feasible, and reproducible. The postoperative surgical outcomes are comparable to the standard RDP 
with the single-console da Vinci Surgical System ${ }^{\circledR}$. This surgical technique can widely improve the robotic surgical training program. Further studies are required to standardize the surgical technique and the robotic surgical training program.

Funding Open access funding provided by Università degli Studi di Verona within the CRUI-CARE Agreement. This research did not receive any specific grant from funding agencies in the public, commercial, or not-for-profit sectors.

\section{Declaration}

Conflict of interest The authors declare that they have no conflict of interest.

Informed consent Informed consent was obtained from all individual participants included in the study.

Open Access This article is licensed under a Creative Commons Attribution 4.0 International License, which permits use, sharing, adaptation, distribution and reproduction in any medium or format, as long as you give appropriate credit to the original author(s) and the source, provide a link to the Creative Commons licence, and indicate if changes were made. The images or other third party material in this article are included in the article's Creative Commons licence, unless indicated otherwise in a credit line to the material. If material is not included in the article's Creative Commons licence and your intended use is not permitted by statutory regulation or exceeds the permitted use, you will need to obtain permission directly from the copyright holder. To view a copy of this licence, visit http://creativecommons. org/licenses/by/4.0/.

\section{References}

1. Venkat R, Edil BH, Schulick RD et al (2012) Laparoscopic distal pancreatectomy is associated with significantly less overall morbidity compared to the open technique: a systematic review and meta-analysis. Ann Surg 255:1048-1059. https://doi.org/10. 1097/SLA.0b013e318251ee09

2. Magge D, Gooding W, Choudry H et al (2013) Comparative effectiveness of minimally invasive and open distal pancreatectomy for ductal adenocarcinoma. JAMA Surg 148:525-531. https://doi.org/10.1001/jamasurg.2013.1673

3. Esposito A, Balduzzi A, De Pastena M et al (2019) Minimally invasive surgery for pancreatic cancer. Expert Rev Anticancer Ther. https://doi.org/10.1080/14737140.2019.1685878

4. Jusoh AC, Ammori BJ (2012) Laparoscopic versus open distal pancreatectomy: a systematic review of comparative studies. Surg Endosc 26:904-913. https://doi.org/10.1007/s00464-0112016-3

5. Nigri GR, Rosman AS, Petrucciani N et al (2011) Metaanalysis of trials comparing minimally invasive and open distal pancreatectomies. Surg Endosc 25:1642-1651. https://doi.org/10.1007/ s00464-010-1456-5

6. Giulianotti PC, Sbrana F, Bianco FM et al (2010) Robot-assisted laparoscopic pancreatic surgery: single-surgeon experience. Surg Endosc 24:1646-1657. https://doi.org/10.1007/s00464-009-08254
7. Zeh HJ, Bartlett DL, Moser AJ (2011) Robotic-assisted major pancreatic resection. Adv Surg 45:323-340

8. Zhou J-Y, Xin C, Mou Y-P et al (2016) Robotic versus laparoscopic distal pancreatectomy: a meta-analysis of short-term outcomes. PLoS ONE. https://doi.org/10.1371/journal.pone.0151189

9. Bolger JC, Broe MP, Zarog MA et al (2017) Initial experience with a dual-console robotic-assisted platform for training in colorectal surgery. Tech Coloproctol. https://doi.org/10.1007/ s10151-017-1687-8

10. Fernandes E, Elli E, Giulianotti P (2014) The role of the dual console in robotic surgical training. Surg 155:1-4. https://doi.org/ 10.1016/j.surg.2013.06.023

11. von Elm E, Altman DG, Egger M et al (2007) the strengthening the reporting of observational studies in epidemiology (STROBE) statement: guidelines for reporting observational studies. Lancet Lond Engl 370:1453-1457. https://doi.org/10.1016/S01406736(07)61602-X

12. Agha R, Abdall-Razak A, Crossley E et al (2019) STROCSS 2019 guideline: strengthening the reporting of cohort studies in surgery. Int J Surg Lond Engl 72:156-165. https://doi.org/10. 1016/j.ijsu.2019.11.002

13. De Pastena M, Esposito A, Paiella S et al (2020) Cost-effectiveness and quality of life analysis of laparoscopic and robotic distal pancreatectomy: a propensity score-matched study. Surg Endosc. https://doi.org/10.1007/s00464-020-07528-1

14. Esposito A, Casetti L, De Pastena M et al (2020) Robotic spleenpreserving distal pancreatectomy: the Verona experience. Updat Surg. https://doi.org/10.1007/s13304-020-00731-8

15. Dindo D, Demartines N, Clavien P-A (2004) Classification of surgical complications. Ann Surg 240:205-213. https://doi.org/ 10.1097/01.sla.0000133083.54934.ae

16. Bassi C, Marchegiani G, Dervenis C et al (2017) The 2016 update of the International study group (ISGPS) definition and grading of postoperative pancreatic fistula: 11 years after. Surgery 161:584-591. https://doi.org/10.1016/j.surg.2016.11.014

17. Pulvirenti A, Landoni L, Borin A et al (2019) Reinforced stapler versus ultrasonic dissector for pancreatic transection and stump closure for distal pancreatectomy: a propensity matched analysis. Surg. https://doi.org/10.1016/j.surg.2019.02.016

18. Bassi C, Molinari E, Malleo G et al (2010) Early versus late drain removal after standard pancreatic resections: results of a prospective randomized trial. Ann Surg. https://doi.org/10.1097/ SLA.0b013e3181e61e88

19. Napoli N, Kauffmann EF, Perrone VG et al (2015) The learning curve in robotic distal pancreatectomy. Updat Surg 67:257-264. https://doi.org/10.1007/s13304-015-0299-y

20. Shakir M, Boone BA, Polanco PM et al (2015) The learning curve for robotic distal pancreatectomy: an analysis of outcomes of the first 100 consecutive cases at a high-volume pancreatic centre. HPB 17:580-586. https://doi.org/10.1111/hpb.12412

21. Shyr B-U, Chen S-C, Shyr Y-M, Wang S-E (2018) Learning curves for robotic pancreatic surgery-from distal pancreatectomy to pancreaticoduodenectomy. Med (Baltim). https://doi.org/10. 1097/MD.0000000000013000

22. Beane M (2019) Shadow learning: building robotic surgical skill when approved means fail - https://journals.sagepub.com/doi/full/ https://doi.org/10.1177/0001839217751692. Accessed 17 May 2020

23. Yuh B, Yu X, Raytis J et al (2016) Use of a mobile tower-based robot-The initial Xi robot experience in surgical oncology. J Surg Oncol 113:5-7. https://doi.org/10.1002/jso.24094

24. Nota CL, Zwart MJ, Fong Y et al (2017) Developing a robotic pancreas program the Dutch experience. J Vis Surg. https://doi. org/10.21037/jovs.2017.07.02

25. Smith AL, Krivak TC, Scott EM et al (2012) Dual-console robotic surgery compared to laparoscopic surgery with respect to 
surgical outcomes in a gynecologic oncology fellowship program. Gynecol Oncol. https://doi.org/10.1016/j.ygyno.2012.05. 017

26. Morgan MSC, Shakir NA, Garcia-Gil M et al (2015) Singleversus dual-console robot-assisted radical prostatectomy: impact on intraoperative and postoperative outcomes in a teaching institution. World J Urol 33:781-786. https://doi.org/10.1007/ s00345-014-1349-7

Publisher's Note Springer Nature remains neutral with regard to jurisdictional claims in published maps and institutional affiliations. 